\title{
The effects of depth and diet on red abalone growth and survival in cage mariculture at San Jeronimo Island, Baja California, Mexico
}

\section{Efectos de la profundidad y la dieta en el crecimiento y la supervivencia del abulón rojo en jaulas de maricultura en la isla San Jerónimo, Baja California, México}

\author{
Jeremie Bauer ${ }^{1}$, Julio Lorda ${ }^{2,3 *}$, Rodrigo Beas-Luna ${ }^{1 *}$, Luis Malpica-Cruz ${ }^{4,5}$, Fabiola Lafarga-De la Cruz ${ }^{6}$, \\ Fiorenza Micheli ${ }^{7}$, Ricardo Searcy-Bernal ${ }^{4}$, Laura Rogers-Bennett ${ }^{8}$, Miguel Bracamontes-Peralta ${ }^{9}$ \\ ${ }^{1}$ Facultad de Ciencias Marinas, Universidad Autónoma de Baja California, Carretera Ensenada-Tijuana, \\ No. 3917, Fraccionamiento Playitas, CP 22860, Ensenada, Baja California, Mexico. \\ 2 Facultad de Ciencias, Universidad Autónoma de Baja California, Carretera Ensenada-Tijuana, No. 3917, Fraccionamiento \\ Playitas, CP 22860, Ensenada, Baja California, Mexico. \\ 3 Tijuana River National Estuarine Research Reserve, 301 Caspian Way, Imperial Beach, California 91932, USA. \\ 4 Instituto de Investigaciones Oceanológicas, Universidad Autónoma de Baja California, Carretera Ensenada-Tijuana, \\ No. 3917, Fraccionamiento Playitas, CP 22860, Ensenada, Baja California, Mexico. \\ 5 ECOCIMATI, Av. del Puerto 2270, Colonia Hidalgo, CP 22880, Ensenada, Baja California, Mexico. \\ 6 Departamento de Acuicultura, Centro de Investigación Científica y de Educación Superior de Ensenada, Carretera \\ Ensenada-Tijuana, No. 3918, Zona Playitas, CP 22860, Ensenada, Baja California, Mexico. \\ ${ }^{7}$ Hopkins Marine Station and Stanford Center for Ocean Solutions, Stanford University, Pacific Grove, California 93950, \\ USA. \\ ${ }^{8}$ Karen C. Drayer Wildlife Health Center and Bodega Marine Laboratory, University of California, Davis, PO Box 247, \\ Bodega Bay, California 94923, USA. \\ 9 Sociedad Cooperativa de Producción Pesquera Ensenada, El Rosario, Baja California, Mexico.
}

* Corresponding authors. E-mails: JL, jlorda@uabc.edu.mx; RBL, rbeas@uabc.edu.mx

\begin{abstract}
In recent decades, global abalone aquaculture has significantly increased, while wild abalone fishery landings have decreased drastically, shifting production from fishing to farming. In California (USA) and Baja California (Mexico), overfishing and climate changelinked diseases are thought to be responsible for mass mortalities and significant declines in abalone fisheries landings. Conservation aquaculture is an option for enhancing abalone populations through captive propagation and cage mariculture with subsequent restockings into the wild. To test, inform, and promote innovative sustainable seafood production strategies in the Northeastern Pacific, we designed an experimental mariculture system at San Jeronimo Island, Baja California. We explored the feasibility of rearing juvenile red abalone, Haliotis rufescens, in a near-shore mariculture cage-based production system to supply individuals for local restoration programs. We tested the effects of 2 different depths, surface and bottom $(5 \mathrm{~m}$ ), and 3 macroalgal diets (Macrocystis pyrifera, Eisenia arborea, and a mixed diet of Pelagophycus porra with M. pyrifera) on the survival and growth of juvenile red abalone ( $32 \pm 3.33 \mathrm{~mm}$ in shell length) inside cages attached to a long-line system. Over the 90 -d experiment, survival was $99 \%$ for the surface treatment and $95 \%$ for the bottom treatment. Mean daily increment in shell length was $93 \pm 12 \mu \mathrm{m} \cdot \mathrm{d}^{-1}$ in surface cages and $82 \pm 13 \mu \mathrm{m} \cdot \mathrm{d}^{-1}$ in bottom cages. Depth did not affect growth or survival. Growth was highest using the E. arborea diet $\left(99 \pm 7 \mu \mathrm{m} \cdot \mathrm{d}^{-1}\right)$ but not significantly different from the $M$. pyrifera and mixed diets $\left(88 \pm 10\right.$ and $74 \pm 13 \mu \mathrm{m} \cdot \mathrm{d}^{-1}$, respectively). High survivorship and growth indicate that San Jeronimo Island can support cage-based mariculture of red abalone and that this strategy may be a useful tool in developing climate-resilient abalone restoration solutions aimed at bolstering seafood production.
\end{abstract}

Key words: conservation aquaculture, Haliotis rufescens, climate change, restoration, macroalgae.

RESUMEN. En décadas recientes, el aumento significativo de la acuicultura global del abulón y la disminución drástica de desembarques de abulón silvestre han ido cambiando la producción de la pesca al cultivo. En California (EUA) y Baja California (México) se cree que enfermedades asociadas al cambio climático y la sobrepesca son la causa de eventos de mortalidad masiva y el declive significativo en los desembarques de abulón silvestre. La acuicultura de conservación es una opción para mejorar las poblaciones de abulón mediante la propagación de organismos de criadero y la maricultura en jaulas, con el subsecuente repoblamiento en la naturaleza. Para experimentar, informar y promover estrategias innovadoras en la producción sostenible de recursos marinos en el Pacífico Nororiental, hemos diseñado un sistema experimental de maricultura en isla San Jerónimo, Baja California. Exploramos la factibilidad de cultivar abulón rojo juvenil, Haliotis rufescens, en un sistema de producción de maricultura costera basado en jaulas, para suplir individuos para programas locales de repoblamiento. Analizamos el efecto de 2 profundidades diferentes, superficie y fondo (5 m), y de 3 dietas de macroalgas (Macrocystis pyrifera, Eisenia arborea, y dieta mixta de Pelagophjycus porra y M. pyrifera $)$ en la supervivencia y el crecimiento de juveniles de abulón rojo ( $32 \pm 3.33 \mathrm{~mm}$ en longitud de concha) dentro de jaulas aseguradas a un sistema de línea madre. Durante el experimento de 90 d, la supervivencia fue del $99 \%$ para el tratamiento de superficie y del $95 \%$ para el tratamiento de fondo. El incremento diario en longitud de concha fue $93 \pm 12 \mu \mathrm{m} \cdot \mathrm{d}^{-1}$ en las jaulas de superficie y $82 \pm 13 \mu \mathrm{m} \cdot \mathrm{d}^{-1}$ en las jaulas de fondo. La profundidad no afectó el crecimiento o la supervivencia. El crecimiento fue mayor con la dieta de $E$. arborea $\left(99 \pm 7 \mu \mathrm{m} \cdot \mathrm{d}^{-1}\right)$, pero no fue significativamente diferente del crecimiento con la dieta de $M$. pyrifera y la dieta mixta $\left(88 \pm 10\right.$ y $74 \pm 13 \mu \mathrm{m} \cdot \mathrm{d}^{-1}$, respectivamente). La alta supervivencia y crecimiento indican que isla San Jerónimo puede sostener la maricultura de abulón rojo en jaulas y que esta estrategia puede ser una herramienta útil para desarrollar soluciones climático-resilientes de restauración de abulón destinadas a impulsar la producción de mariscos.

Palabras clave: acuicultura de conservación, Haliotis rufescens, cambio climático, restauración, macroalgas. 


\section{INTRODUCTION}

Given the multiple environmental and anthropogenic stressors, the current worldwide abalone production has dramatically changed from fishing to farming. Wild abalone fisheries landings have decreased significantly in the past decades, from 20,000 metric tons (mt) in the 1970s to about 6,500 mt in 2016/2017 (Cook 2019), whereas production of farmed abalone has increased from $50 \mathrm{mt}$ in the 1970 s to $160,987 \mathrm{mt}$ in 2016/2017 (Cook 2016, 2019). Aquaculture has served as a promising tool to reduce the impact of fisheries and has been highlighted as a potential solution to food availability and security in the United Nations 2030 Agenda (UN-DESA 2018). However, there is a paucity of scientific information on ensuring a robust and sustainable aquaculture regime in the face of a changing climate. Rising sea surface temperatures and the increased frequency and severity of storms are stressors to mariculture worldwide (Barange and Perry 2009) and particularly detrimental to developing abalone aquaculture industries in countries like Mexico.

Wild abalone populations have declined in Mexico and recovery is now uncertain owing to climate change impacts. Although the worldwide trend is an increase in abalone aquaculture, in Mexico the wild abalone fisheries still produce 10 times more than the abalone aquaculture industry (300 vs. $23.5 \mathrm{mt} \cdot \mathrm{y}^{-1}$, respectively; Cook 2019). The abalone fishery in Mexico takes place on the Pacific coast of the Baja California Peninsula, where it continues to be economically important despite the fact that landings in recent years are only at $5 \%$ of the historical maximum (Morales-Bojórquez et al. 2008; Guzmán-Del Próo et al. 2013, 2017). Fishing in the northeastern Pacific, including Baja California, has deteriorated because of a combination of multiple stressors, such as overfishing, diseases, extreme warming events, and hypoxia (Lafferty and Kuris 1993, Karpov et al. 2000, Micheli et al. 2012, Ben-Horin et al. 2016, Boch et al. 2018, Lonhart et al. 2019). For example, in northern California, wild abalone populations have been affected by extreme warming events combined with other stressors, which caused mass mortalities of red abalone leading to the closure of the fishery in 2018 (Rogers-Bennett and Catton 2019). It is crucial to increase aquaculture production, as it has been shown around the world to support the local fishery both ecologically and economically.

Additional conservation and management actions are needed for the recovery of natural populations and sustainable fisheries in the northern portion of the Baja California Peninsula. Shifts in oceanic conditions have resulted in landings of less than $100 \mathrm{mt}$ from 2012 up until 2017 (DOF 2018). The Mexican government highlights that aquaculture is a potential strategy to increase abalone production (DOF 2018). Therefore, an opportunity exists to develop abalone aquaculture programs in Mexico that might help support conservation programs.

\section{INTRODUCCIÓN}

Actualmente, debido a múltiples fuentes de estrés ambiental y antropogénicas, la producción mundial de abulón ha migrado drásticamente de la pesca al cultivo. Los desembarques de abulón silvestre han disminuido significativamente en las últimas décadas, de 20,000 toneladas métricas (tm) en la década de 1970 a aproximadamente 6,500 tm en 2016/2017 (Cook 2019), mientras que la producción de abulón cultivado ha incrementado de $50 \mathrm{tm}$ en la década de 1970 a 160,987 tm en 2016/2017 (Cook 2016, 2019). La acuicultura es una herramienta prometedora para reducir el impacto de la pesca y ha sido resaltada en la Agenda 2030 de las Naciones Unidas como una solución potencial para problemas de disponibilidad de comida y seguridad alimentaria (UN-DESA 2018). Sin embargo, existe una escasez de información científica sobre el aseguramiento de regímenes de acuicultura robustos y sostenibles en presencia del cambio climático. El incremento de la temperatura superficial del mar y el aumento en la frecuencia y severidad de las tormentas son estresores que afectan mundialmente a la maricultura (Barange y Perry 2009), y son particularmente perjudiciales para las industrias de acuicultura de abulón en desarrollo en países como México.

Las poblaciones de abulón silvestre en México han declinado, y es incierta su recuperación debido a los impactos del cambio climático. Aunque la tendencia mundial es un incremento en la acuicultura de abulón, en México las pesquerías de abulón silvestre todavía producen 10 veces más que su industria acuícola (300 vs. $23.5 \mathrm{tm}$ al año, respectivamente; Cook 2019). En México, la pesquería de abulón se lleva a cabo en la costa del Pacífico de la península de Baja California, donde continúa siendo económicamente importante a pesar de que los desembarques en años recientes son tan sólo un 5\% del máximo histórico (Morales-Bojórquez et al. 2008; Guzmán-Del Próo et al. 2013, 2017). Este deterioro en la pesca en Baja California, y en el resto del Pacífico Nororiental, se debe a la combinación de múltiples estresores, tales como la sobrepesca, enfermedades, eventos de calentamiento e hipoxia (Lafferty y Kuris 1993, Karpov et al. 2000, Micheli et al. 2012, Ben-Horin et al. 2016, Boch et al. 2018, Lonhart et al. 2019). En el Norte de California, EUA, por ejemplo, las poblaciones de abulón silvestre han sido afectadas por ondas extremas de calor combinadas con otros estresores, lo cual causó mortalidades masivas de abulón rojo que llevaron al cierre de la pesquería en 2018 (Rogers-Bennett y Catton 2019). Es crucial el incremento de la producción acuícola, ya que se ha demostrado alrededor del mundo que esto apoya a las pesquerías locales tanto ecológica como económicamente.

Acciones adicionales de manejo y conservación son necesarias para la pesquería sostenible y la recuperación de las poblaciones silvestres en el norte de la península de Baja California. Cambios en condiciones oceánicas han resultado en capturas de menos de 100 tm desde el año 2012 a 2017 
In Ensenada, Baja California, red abalone mariculture (ocean-based efforts) has been carried out in the past with different techniques and macroalgae diets. In 1990, Searcy-Bernal and Salas-Garza (1990) conducted the first experimental mariculture project to grow red abalone, Haliotis rufescens, with a diet of giant kelp, Macrocystis pyrifera, and feather boa kelp, Egregia menziesii, in floating cages in Todos Santos Bay. At about the same time, a commercial red abalone mariculture company called "Abulones Cultivados" was established near Todos Santos Island, using cages and M. pyrifera as the main feed. However, the company moved to land-based facilities (Searcy-Bernal et al. 2010) and as of 2020 is still operating in the area of Ejido Erendira. More recently, Zertuche-González et al. (2014) evaluated the growth performance of red abalone in an integrated multi-trophic aquaculture farm for one year in San Quintin Bay. Their study demonstrated the feasibility of growing red abalone juveniles at sea with a diet of Eisenia arborea. These studies have laid the basis for the development of abalone mariculture in the region, but it is important to continue experiments to cope with environmental changes associated with climate change.

Since the early mariculture efforts in Baja California, the understanding of local oceanographic and circulation patterns, along with mariculture technology, has improved. Furthermore, the ongoing declines of wild abalone have altered the attitudes and perceptions of local fishing cooperatives towards aquaculture as a potential solution. Efforts to enhance the wild populations are now supported by those economically linked to abalone fisheries. Mariculture could promote production of abalone for outplanting in local reefs and promote conservation aquaculture to restore natural populations (Froehlich et al. 2017, 2018). Nevertheless, the changing climate generates new challenges that are particularly affecting the availability of macroalgae, a fundamental resource for the abalone aquaculture industry.

The availability of macroalgae, the main food for abalone, is crucial for the development of the aquaculture industry. In the northeastern Pacific, abalone farms in Mexico and the USA typically use giant kelp, $M$. pyrifera, harvested from the wild as feed (Evans and Langdon 2000, Garcia-Esquivel and Felbeck 2009). However, in the last 2 decades extreme events such as ENSO and marine heatwaves have substantially affected giant kelp populations in Baja California (Ladah et al. 1999; Arafeh-Dalmau et al. 2019, 2020; Cavanaugh et al. 2019; Beas-Luna et al. 2020). Because of the recent variability in giant kelp biomass availability, it is important to assess other macroalgae as alternative food sources for abalone mariculture. For example, the southern sea palm kelp, E. arborea, is capable of surviving in relatively warmer waters $\left(>20^{\circ} \mathrm{C}\right)$ with low nutrients, where other kelps cannot survive (Hernández-Carmona et al. 2000, 2001; Zertuche-González et al. 2014). Therefore, E. arborea or other kelp species, such as Pelagophycus porra, may potentially provide a food supply to aquaculture operations
(DOF 2018). El gobierno mexicano destaca a la acuicultura como una estrategia potencial para incrementar la producción de abulón (DOF 2018), lo que genera una oportunidad para desarrollar programas de acuicultura de abulón en México que puedan apoyar a programas de conservación.

En Ensenada, Baja California, se han empleado diversas técnicas y dietas a base de macroalgas para llevar a cabo la maricultura (cultivos en el océano) de abulón rojo. En 1990, Searcy-Bernal y Salas-Garza (1990) condujeron el primer proyecto experimental de maricultura para criar abulón rojo, Haliotis rufescens, basado en una dieta de sargazo gigante, Macrocystis pyrifera, y cola de zorro, Egregia menziesii, en jaulas flotantes en la bahía de Todos Santos. Aproximadamente al mismo tiempo, una compañía de maricultura comercial de abulón rojo llamada "Abulones Cultivados" se estableció cerca de isla Todos Santos, la cual utilizó jaulas y M. pyrifera como alimento principal. Sin embargo, la compañía fue trasladada a instalaciones terrestres (Searcy-Bernal et al. 2010) y, hasta la fecha, 2020, opera en el área de ejido Eréndira. Más recientemente, ZertucheGonzález et al. (2014) evaluaron el crecimiento del abulón rojo en una granja de acuicultura multitrófica integrada por un año en la bahía de San Quintín. Este estudio demostró la factibilidad de criar juveniles de abulón rojo en campo con una dieta a base de la macroalga coliflor, Eisenia arborea. Estos estudios han sentado las bases para el desarrollo de la maricultura de abulón en la región, pero es importante continuar con experimentos para encontrar formas de lidiar con cambios ambientales asociados al cambio climático.

Desde estos primeros esfuerzos de maricultura en Baja California, la comprensión de patrones locales oceanográficos y de circulación y la tecnología de maricultura han mejorado. Adicionalmente, el declive continuo de las poblaciones de abulón silvestre ha modificado las actitudes y percepciones de las cooperativas pesqueras locales hacia la acuicultura como una potencial solución. Los actores económicamente ligados a la pesquería de abulón ahora apoyan los esfuerzos para mejorar las poblaciones silvestres. La maricultura podría promover la producción de abulón para el trasplante en arrecifes locales y promover la acuicultura de conservación para restaurar poblaciones silvestres (Froelich et al. 2017, 2018). Sin embargo, el cambio climático genera nuevos desafíos que, en particular, están afectando la disponibilidad de macroalgas, un recurso fundamental para la industria acuícola del abulón.

La disponibilidad de macroalgas, el alimento principal del abulón, es crucial para el desarrollo de la industria acuícola. En el Pacífico Nororiental, las granjas de abulón en México y Estados Unidos suelen utilizar el sargazo gigante, M. pyrifera, extraído del medio natural, como alimento (Evans y Langdon 2000, Garcia-Esquivel y Felbeck 2009). Sin embargo, en las últimas 2 décadas, eventos extremos, tales como El Niño y ondas de calor marinas, han afectado negativamente a las poblaciones de sargazo gigante en Baja California (Ladah et al. 1999; Arafeh-Dalmau et al. 2019, 
during times of low giant kelp biomass. Extreme events not only affect macroalgae availability but also present challenges to abalone physiology and mariculture structures.

Increased frequency and intensity of storms and heatwave events can negatively affect shallow mariculture systems. An option to potentially ameliorate the detrimental effects of these issues is to move the cages deeper in the water column to avoid warm surficial waters and to minimize storm-induced damage via intense wave action and surge. As the frequency, duration, and intensity of marine heat waves increases in the coming decades (Oliver et al. 2018), finding depth refuges from warm water and alternatives to giant kelp resources is of utmost importance for the future of abalone production. To test the potential for abalone mariculture in San Jeronimo Island, Baja California, Mexico, under current conditions we explored the feasibility of growing red abalone in cages attached to a vertical long-line system. Specifically, we tested for the effects of depth (surface and bottom at $5 \mathrm{~m}$ ) and 3 different brown macroalgal diets on the growth and survival of red abalone juveniles.

\section{MATERIALS AND METHOdS}

\section{Experimental study area}

This study was carried out at San Jeronimo Island $(1.3 \mathrm{~km}$ long and $500 \mathrm{~m}$ wide), located in the Ensenada Municipality in the state of Baja California, Mexico (Fig. 1; 29 $47^{\prime} 34.9^{\prime \prime}$ $\left.\mathrm{N}, 115^{\circ} 47^{\prime} 31.9^{\prime \prime} \mathrm{W}\right)$. The marine resources of this area are under the jurisdiction of the fishing cooperative "Sociedad Cooperativa de Producción Pesquera Ensenada, S.C.L.", which participated in this study. Juvenile red abalone were provided by the commercial aquaculture farm "Abulones Cultivados", which is $250 \mathrm{~km}$ north of the study area. Juvenile abalone (ca. $30 \pm 1 \mathrm{~mm}$ ) were transported from the aquaculture farm to San Jeronimo Island in coolers with a wet sponge base. After arriving, 2,100 juvenile abalone were immediately transferred to the experimental cages and fed ad libitum fresh $M$. pyrifera for a month prior to initiating the experiment. Average sea surface temperature during this period was $14.98 \pm 0.74{ }^{\circ} \mathrm{C}$.

\section{Experimental design}

An experimental mariculture system was designed to assess the effect of different rearing conditions (depth and diet) on the growth and survival of abalone. The outplanting site was a maximum of $5 \mathrm{~m}$ deep, had a sandy bottom, and was located on the protected southeast region of the island. The experimental mariculture system consisted of cages attached to a line at 2 depths (Fig. 2). Two anchors (iron pipes $7.5 \mathrm{~cm}$ in diameter and $1.5 \mathrm{~m}$ long) were buried in the seabed at an angle of approximately $45^{\circ}$. Buoys (200-L drums with pressurized air) were attached to the anchors and distributed along the $100-\mathrm{m}$ long float line (polypropylene
2020; Cavanaugh et al. 2019; Beas-Luna et al. 2020). Debido a la reciente variabilidad en la disponibilidad de biomasa de sargazo gigante, es de suma importancia evaluar otras macroalgas como fuentes alternativas de comida para la maricultura de abulón. Por ejemplo, la coliflor, E. arborea, es capaz de sobrevivir en aguas relativamente más cálidas $\left(>20{ }^{\circ} \mathrm{C}\right)$ y de bajos nutrientes, donde otras macroalgas no logran sobrevivir (Hernández-Carmona et al. 2000, 2001; ZertucheGonzález et al. 2014). Por tanto, E. arborea u otras especies de macroalgas, tales como Pelagophycus porra, podrían tener el potencial de proveer un suministro de alimentos para las operaciones acuícolas durante épocas de baja disponibilidad de biomasa de sargazo gigante. Los eventos extremos no sólo afectan la disponibilidad de macroalgas, sino que presentan desafíos a la fisiología de los abulones y a las estructuras de maricultura.

El incremento en la frecuencia y la intensidad de tormentas y eventos de ondas de calor puede afectar negativamente a sistemas de maricultura someros. Una opción para reducir estos efectos es mover las jaulas a mayor profundidad en la columna de agua, con el fin de evitar las aguas cálidas superficiales y minimizar los daños inducidos por la intensa acción del oleaje durante tormentas. Mientras la frecuencia, duración e intensidad de las ondas de calor sigan incrementando en las próximas décadas (Oliver et al. 2018), será de suma importancia para el futuro de la producción de abulón hallar refugios de las aguas cálidas en aguas profundas y alternativas al sargazo gigante como alimento. Para examinar el potencial de la maricultura en isla San Jerónimo, Baja California, México, bajo las condiciones actuales, exploramos la factibilidad de cultivar abulón rojo en jaulas aseguradas a un sistema de línea madre vertical. Específicamente, examinamos el efecto de la profundidad (en superficie y en el fondo a $5 \mathrm{~m}$ ) y el efecto de dietas a base de 3 macroalgas diferentes en el crecimiento y la supervivencia de juveniles de abulón rojo.

\section{MATERIALES Y MÉTODOS}

\section{Área de estudio}

Este estudio fue llevado a cabo en isla San Jerónimo (1.3 km de largo y $500 \mathrm{~m}$ de ancho), la cual se encuentra en la municipalidad de Ensenada, en el estado de Baja California, México (Fig. 1; 2947'34.9" N, 115²7'31.9" W). Los recursos marinos de esta área están bajo la jurisdicción de la cooperativa pesquera "Sociedad Cooperativa de Producción Pesquera Ensenada, S.C.L.”, la cual participó en este estudio. Los abulones rojos juveniles utilizados fueron provistos por la granja acuícola "Abulones Cultivados", la cual se encuentra a $250 \mathrm{~km}$ al norte del área de estudio. Los abulones juveniles (ca. $30 \pm 1 \mathrm{~mm}$ ) fueron transportados de las instalaciones acuícolas a isla San Jerónimo en hieleras con un fondo de esponja húmeda. Al llegar, 2,100 juveniles de abulón rojo fueron inmediatamente transferidos a las jaulas 
rope). Cages consisted of plastic-coated metal mesh $(0.80 \times$ $0.80 \times 0.36 \mathrm{~m})$, which was then wrapped with $0.5-\mathrm{cm}$ plastic "Vexar" mesh. Eight cages were attached to the float line, 4 suspended near the surface and 4 on the sandy seafloor $(5 \mathrm{~m}$ depth) weighted with rocks in each corner of the cage. Each cage contained 3 Australian baskets $(0.75 \times 0.25 \times 0.20 \mathrm{~m})$ stacked on one another (Fig. 2). Additionally, to increase the available surface for the abalone, Australian baskets were each modified at the center with a plastic plate with holes, allowing abalone to move throughout the basket. One HOBO U22 Temp Pro V2 temperature sensor was installed in each cage, and temperature $\left({ }^{\circ} \mathrm{C}\right)$ was recorded every half hour throughout the experiment.

The 2,100 juvenile red abalone were randomly placed into the 24 modified Australian baskets. Each basket had between 87 and 104 abalone, with a density of 102-122 abalone per square meter, as recommended by Viera et al. (2014). Fifteen abalone per Australian basket (360 individuals, 17\% of the 2,100 animals) were tagged with shellfish tags (Floy Tag and Mfg.) and measured at the start of the experiment. It was not possible to tag more animals because of logistic and economic constraints. The mean initial shell length of these tagged abalone was $32 \pm 3.33 \mathrm{~mm}$ (mean $\pm \mathrm{SD}$ ).

In addition to the effect of depth (surface $v s .5 \mathrm{~m}$ ), the effect of macroalgal diet on abalone growth parameters was evaluated. Considering the most abundant brown macroalgae around the mariculture area, red abalone were fed 1 of 3 different diets. The first diet tested was $100 \%$ giant kelp, M. pyrifera, the second diet was $100 \%$ southern palm kelp, $E$. arborea, and the third diet was a mix of $50 \%$ elk kelp, P. porra, and $50 \% \mathrm{M}$. pyrifera. All diets consisted of kelp

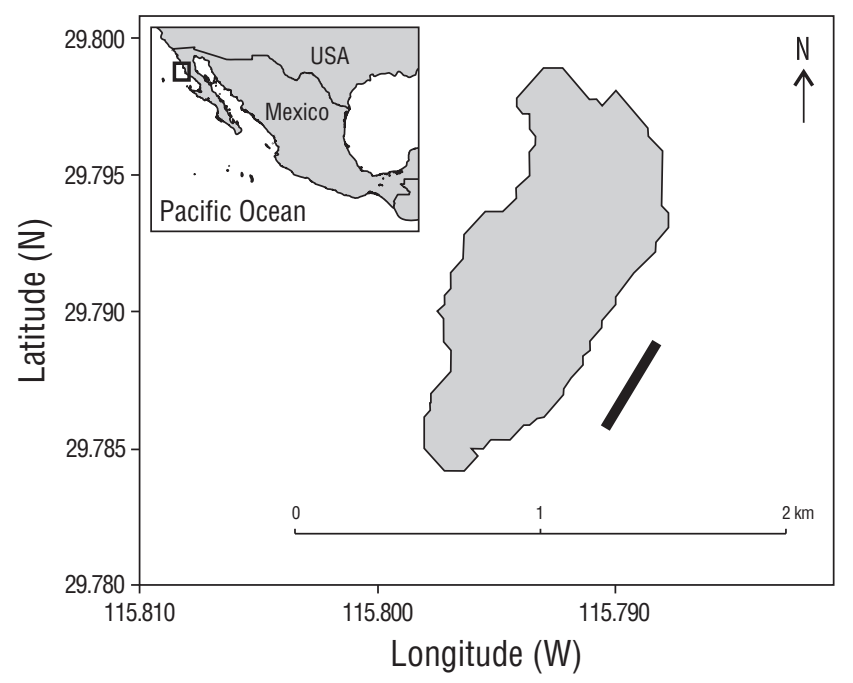

Figure 1. Experimental study area, San Jeronimo Island, Ensenada, Baja California, Mexico. The black bar indicates the mariculture zone.

Figura 1. Área del estudio, isla San Jerónimo, Ensenada, Baja California, México. La barra negra indica el área de maricultura. experimentales y alimentados con $M$. pyrifera ad libitum por un mes previo a iniciar el experimento. La temperatura superficial del mar promedio durante este periodo fue de $14.98 \pm 0.74{ }^{\circ} \mathrm{C}$.

\section{Diseño experimental}

Se diseñó un sistema experimental de maricultura con el fin de evaluar el efecto de diferentes condiciones de crianza (profundidad y dieta) en el crecimiento y la supervivencia de los abulones. El sitio de cultivo elegido presentó una profundidad máxima de $5 \mathrm{~m}$, con suelo arenoso, y se ubicó en la región protegida al sureste de la isla. El sistema experimental consistió en una línea madre con jaulas aseguradas a 2 profundidades distintas (Fig. 2). Dos anclas (tubos de hierro con un diámetro de $7.5 \mathrm{~cm}$ y de $1.5 \mathrm{~m}$ de largo) fueron enterradas en el lecho marino con un ángulo aproximado de $45^{\circ}$, y a estas se aseguraron boyas superficiales (tambos de 200 L con aire presurizado) sujetas a líneas de flotación (cuerdas de polipropileno) de $100 \mathrm{~m}$ de largo, distribuidas con boyas adicionales. Las jaulas fueron elaboradas con malla de metal recubierta de plástico $(0.80 \times 0.80 \times 0.36 \mathrm{~m})$ y envueltas con una malla de plástico "Vexar" de $0.5 \mathrm{~cm}$. Ocho jaulas fueron aseguradas a la línea de flotación, 4 a nivel superficial, y otras 4 en el fondo a $5 \mathrm{~m}$, con rocas como pesos en cada esquina de las jaulas. Cada jaula contuvo en $\mathrm{su}$ interior 3 canastas australianas $(0.75 \times 0.25 \times 0.20 \mathrm{~m})$ apiladas (Fig. 2). Adicionalmente, para incrementar la superficie de contacto disponible para los abulones, se modificaron las canastas australianas añadiéndoles una lámina de plástico con agujeros en el medio, lo cual permitió el movimiento de los abulones dentro de la canasta. En cada jaula se instaló un sensor térmico HOBO U22 Temp Pro V2, que registró la temperatura $\left({ }^{\circ} \mathrm{C}\right)$ cada media hora durante todo el experimento.

Los 2,100 juveniles de abulón rojo fueron colocados al azar en las 24 canastas australianas modificadas. Cada canasta contuvo entre 87 y 104 abulones, con una densidad de 102-122 abulones por metro cuadrado, siguiendo las recomendaciones de Viera et al. (2014). Quince abulones por canasta australiana fueron marcados con marcas shellfish (Floy Tag y Mfg.) y medidos al inicio del experimento. Debido a limitaciones logísticas y económicas, no fue posible marcar a más individuos. El largo de concha promedio de los organismos marcados fue de $32 \pm 3.33 \mathrm{~mm}$ (promedio \pm desviación estándar) al inicio.

Además del efecto de la profundidad (superficie vs. $5 \mathrm{~m}$ ), se evaluó el efecto de la dieta de macroalgas en los parámetros de crecimiento de los abulones. Con base en las macroalgas más abundantes alrededor del área de maricultura, se alimentó a los abulones con 3 dietas diferentes. La primera dieta experimental consistió de 100\% sargazo gigante, $M$. pyrifera, la segunda consistió de 100\% coliflor, $E$. arborea, y la tercera consistió de una mezcla de $50 \%$ cuerno de alce, $P$. porra, y 50\% M. pyrifera. Todas 


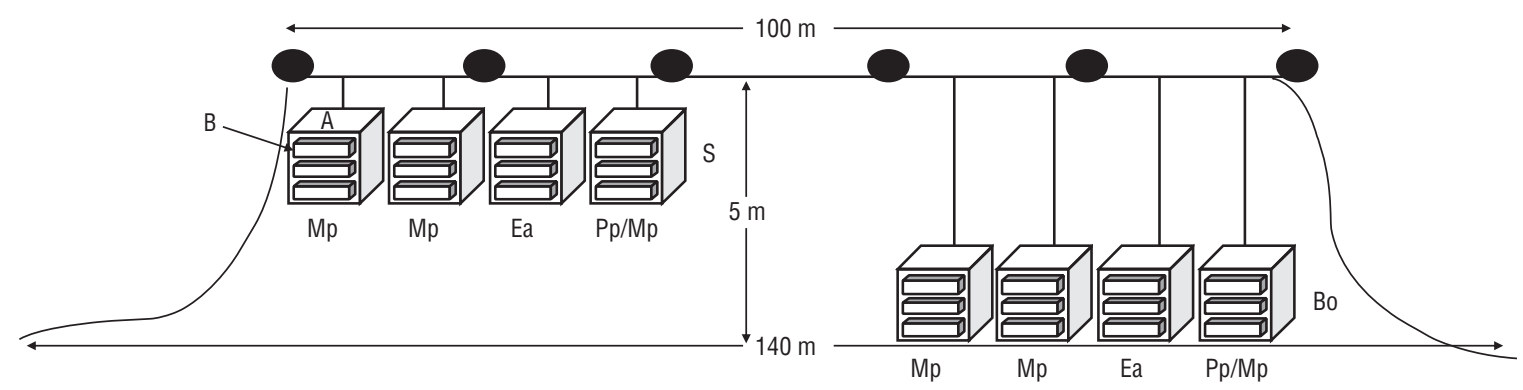

Figure 2. Design of the experimental long-line system. Depth $=5 \mathrm{~m}$; A, metal cage; B, Australian baskets; S, surface treatment; Bo, bottom treatment. Diets: Mp, Macrocystis pyrifera; Ea, Eisenia arborea; $\mathrm{Pp} / \mathrm{Mp}, 50 \%$ Pelagophycus porra/50\% Macrocystis pyrifera. Filled ovals represent buoys along the float line.

Figura 2. Diseño del sistema experimental de línea madre. Profundidad = $5 \mathrm{~m} ; \mathrm{A}$, jaula metálica; $\mathrm{B}$, canastas australianas; $\mathrm{S}$, tratamiento de superficie; Bo, tratamiento profundo. Dietas: Mp, Macrocystis pyrifera; Ea, Eisenia arborea; Mp/Pp, 50\% Pelagophycus porra/50\% Macrocystis pyrifera. Los óvalos rellenos representan boyas a lo largo de la línea de flotación.

blades. Of the 4 surface cages, 2 cages housed M. pyrifera diets, 1 cage the E. arborea diet, and 1 cage the mixed $50 \%$ P. porra $/ 50 \%$ M. pyrifera diet. We repeated this design with the 4 bottom cages. Given the logistic constraints, only 8 cages could be used; therefore, only 1 diet per depth could be replicated, and we decided to do this for the $100 \%$ M. pyrifera diet, which is the most abundant and the standard feed in culture systems in northern Baja California Peninsula. The E. arborea blades were harvested by scuba diving; the canopy blades (upper $3 \mathrm{~m}$ ) of M. pyrifera, by hand from a boat; and the P. porra blades, from algae that had drifted into the mariculture area. Abalone were fed ad libitum once per week, and the excess of macroalgae was removed weekly. The experiment ran for 3 months (90 d), from March to June 2019.

\section{Growth of red abalone}

Shell length of the 360 tagged red abalone was measured at time zero and at days 47 and 90 using a digital caliper (Mitutoyo Absolute AOS CD 6"AX) with $\pm 0.01 \mathrm{~mm}$ accuracy. Means for each cage were calculated from all the animals measured in the 3 baskets inside the cage (experimental unit). The mean for the M. pyrifera treatment was calculated from the 2 replicate cages. Data for the 3 baskets per cage were pooled, since it was not an objective of this study to assess the variability among baskets. These baskets were used to provide protection, larger culture surface, and even distribution of abalone and algae.

Mean shell length of tagged abalone was used to calculate the daily increment in shell length (DISL), monthly growth rate (MGR), and total growth (TG) as reported by Hopkins (1992). Survivorship was assessed weekly during feeding, and dead abalone were removed from the cages.

\section{Statistical analysis}

All statistical analyses were performed with STATISTICA v.12 software. To test for differences in abalone growth las dietas consistieron de láminas de macroalga. Entre las 4 jaulas superficiales, 2 jaulas fueron asignadas una dieta de M. pyrifera y cada una de las 2 jaulas restantes fueron asignadas la dieta de E. arborea y la dieta mixta de $50 \%$ P. porra $/ 50 \%$ M. pyrifera, respectivamente. Repetimos este diseño experimental con las 4 jaulas de fondo. Debido a las restricciones logísticas, sólo se pudieron utilizar estas 8 jaulas, por lo que solamente se pudo replicar una dieta por profundidad. Se decidió replicar la dieta de 100\% M. pyrifera, por ser el alimento estándar y el más abundante en sistemas de cultivo en el norte de la península de Baja California. La cosecha de láminas de E. arborea fue llevada a cabo por medio de buceo autónomo; la de las láminas del dosel (3 m superiores) de M. pyrifera, a mano desde embarcaciones; y la de las láminas de $P$. porra, de individuos flotantes que habían derivado hacia el área de maricultura. Los abulones fueron alimentados ad libitum una vez a la semana, y de igual forma, las macroalgas restantes fueron removidas semanalmente. El experimento duró 3 meses (90 d), de marzo a junio de 2019.

\section{Crecimiento del abulón rojo}

El largo de la concha de los 360 abulones marcados fue medido antes de iniciar el experimento y en el día 47 y el día 90 utilizando un vernier digital (Mitutoyo Absolute AOS CD 6 "AX) con una precisión de $\pm 0.01 \mathrm{~mm}$. El promedio por jaula fue calculado utilizando las medidas de todos los organismos en las 3 canastas dentro de la jaula (unidad experimental). El promedio del tratamiento de $M$. pyrifera fue calculado considerando las 2 réplicas o jaulas . Los datos obtenidos por canasta dentro de las jaulas fueron agrupados, ya que no era un objetivo de este estudio el estimar la variabilidad entre canastas. Estas solo fueron utilizadas para proveer protección, incrementar la superficie para los abulones, y tener una distribución uniforme de abulones y algas.

El promedio de la longitud de concha de los abulones marcados fue utilizada para calcular el incremento diario en 
among treatments, we conducted a two-way analysis of variance (ANOVA) with water depth and diet as fixed factors. This design can be considered as an unbalanced factorial with replication $(n=2)$ for only the $M$. pyrifera diet in both depth treatments since, given the logistic constraints, it was not possible to include replicates for the 2 other diets. Statistical analysis of the main effects is possible, but the interaction term cannot be adequately evaluated. Results must therefore be used cautiously given the lack of replication (Aitkin 1978, Langsrud 2003). We did a posteriori power analysis to find the smallest sample size for the differences to be statistically different between treatments with JMP V.14 software. Levene's $F$-test was used to assess the homogeneity of variances (Brown and Forsythe 1974). Only the analysis for total abalone growth ( $\mathrm{mm}$ ) at the end of the trial is reported, since the analysis for the daily and monthly rates were similar.

\section{RESULTS}

\section{Depth effect on red abalone growth and survival}

When pooling diet means for each depth, abalone had a mean DISL of $93 \pm 12 \mu \mathrm{m} \cdot \mathrm{d}^{-1}$ in the surface cages and $82 \pm$ $13 \mu \mathrm{m} \cdot \mathrm{d}^{-1}$ in the bottom cages. On average, during the experimental period, the abalone from the surface cages grew more than the abalone from the bottom cages, $8.33 \pm 1.06$ vs. $7.38 \pm 1.13 \mathrm{~mm}$ TG, respectively, and $2.8 \pm 0.4$ vs. $2.5 \pm$ $0.4 \mathrm{~mm} \cdot \mathrm{month}^{-1} \mathrm{MGR}$, respectively. These differences were not statistically significant (Fig. 3, Table 1; two-way ANOVA,

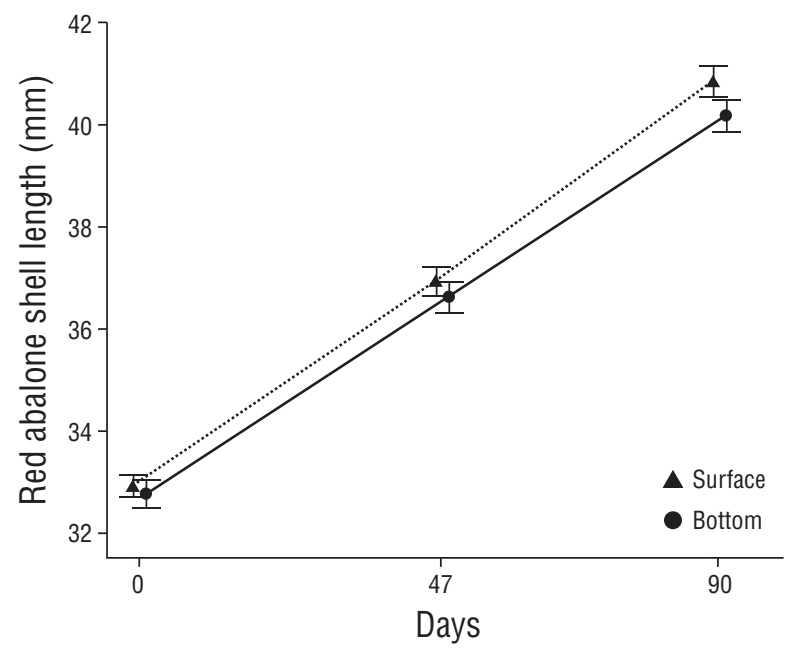

Figure 3. Red abalone, Haliotis rufescens, mean growth in shell length during $90 \mathrm{~d}$ of experiments in 2 depth treatments (two-way analysis of variance, $\left.F_{1,4}=3.066, P=0.155\right)$ : surface and bottom. Vertical bars denote standard error.

Figura 3. Crecimiento promedio en longitud de concha del abulón rojo, Haliotis rufescens, durante $90 \mathrm{~d}$ de experimento en 2 tratamientos de profundidad (análisis de varianza de 2 vías, $\left.F_{1,4}=3.066, P=0.155\right)$ : en superficie y en el fondo. Las barras verticales denotan el error estándar. longitud de concha (IDLC), la tasa de crecimiento mensual (TCM) y el crecimiento total (CT), como en Hopkins (1992). La supervivencia fue estimada semanalmente durante la alimentación, y los abulones muertos fueron removidos de las jaulas.

\section{Análisis estadístico}

Todos los análisis estadísticos fueron realizados con el software STATISTICA v.12. Para examinar las diferencias en el crecimiento de los abulones entre tratamientos, condujimos un análisis de varianza (ANDEVA) de 2 vías con la profundidad y la dieta como variables fijas. Este diseño se puede considerar como factorial no balanceado con replicación $(n=2)$ solamente para la dieta de $M$. pyrifera en ambas profundidades ya que, debido a las limitaciones logísticas, no fue posible incluir replicación para las otras 2 dietas. El análisis estadístico de los efectos principales es posible, pero la interacción no puede ser evaluada, por lo que los resultados se deben tomar con precaución dada la falta de replicación (Aitkin 1978, Langsrud 2003). Realizamos un análisis de poder a posteriori para hallar el tamaño de muestra menor para encontrar diferencias significativas entre los tratamientos con el software JMP V.14. La prueba $F$ de Levene fue utilizada para evaluar la homogeneidad de varianza (Brown y Forsythe 1974). Solo el análisis para la tasa de crecimiento total de los abulones $(\mathrm{mm})$ al final del experimento fue reportado, ya que el análisis para las tasas diarias y mensuales resultaron muy similares.

\section{Resultados}

\section{Efecto de la profundidad en la supervivencia y el crecimiento del abulón rojo}

Al agrupar los promedios de las dietas por profundidad, los abulones tuvieron IDLC de $93 \pm 12 \mu \mathrm{m} \cdot \mathrm{d}^{-1}$ en las jaulas superficiales y de $82 \pm 13 \mu \mathrm{m} \cdot \mathrm{d}^{-1}$ en las jaulas de fondo. En promedio, durante el periodo experimental, los abulones en las jaulas superficiales crecieron más que los abulones en las jaulas profundas, con un CT de $8.33 \pm 1.06$ vs. $7.38 \pm$ $1.13 \mathrm{~mm}$, respectivamente, y una TCM de $2.8 \pm 0.4$ vs. $2.5 \pm$ $0.4 \mathrm{~mm} \cdot \mathrm{mes}^{-1}$, respectivamente. Estas diferencias no fueron estadísticamente significativas (Fig. 3, Tabla 1, ANDEVA de 2 vías, $F_{1,4}=3.066, P=0.155$ ), por lo que hicimos un análisis de poder a posteriori para hallar el tamaño de muestra más pequeño que hubiese sido capaz de hallar una diferencia estadística entre los 2 tratamientos de fondo. Hallamos que, si nuestro diseño experimental hubiese tenido 3 o 4 réplicas por tratamiento, la diferencia entre profundidades en el crecimiento de los organismos podría haber sido estadísticamente significativa $(\alpha=0.05, \sigma=0.77, \delta=0.47): N=18$, poder $=$ $0.69 ;$ y $N=24$, poder $=0.82$, respectivamente. El crecimiento promedio fue de $84 \pm 2 \mu \mathrm{m} \cdot \mathrm{d}^{-1}$ durante el primer periodo de muestreo (del día 0 al 46) y de $91 \pm 12 \mu \mathrm{m} \cdot \mathrm{d}^{-1}$ en el segundo 
Table 1. Statistical information of the two-way analysis of variance performed with the data of total growth in shell length of red abalone under diet and depth treatments during the 90-d experiment. SS, sum of squares; MS, mean square.

Tabla 1. Información estadística del análisis de varianza de 2 vías aplicado a los datos del crecimiento total en el largo de concha del abulón rojo bajo tratamientos de dieta y profundidad durante $90 \mathrm{~d}$ de experimento. SS, suma de cuadrados; MS, media de cuadrados.

\begin{tabular}{lccccc}
\hline & SS & d.f. & MS & $F$ & $P$ \\
\hline Diet & 4.832 & 2 & 2.412 & 4.120 & 0.107 \\
Depth & 1.798 & 1 & 1.798 & 3.066 & 0.155 \\
Error & 2.345 & 4 & 0.586 & & \\
\hline
\end{tabular}

$\left.F_{1,4}=3.066, P=0.155\right)$, so we performed $a$ posteriori power analysis to find the smallest sample size for the differences between the 2 depth treatments to be statistically significant. If our experimental design would have had 3 or 4 replicates per treatment, the difference in growth between depths might have been statistically significant $(\alpha=0.05, \sigma=0.77$, $\delta=0.47): N=18$, power $=0.69$; and $N=24$, power $=0.82$, respectively. Growth was $84 \pm 2 \mu \mathrm{m} \cdot \mathrm{d}^{-1}$ during the first sampling period (days $0-46$ ) and $91 \pm 12 \mu \mathrm{m} \cdot \mathrm{d}^{-1}$ during the second period (days 47-90) (Fig. 3). Average abalone survival was $99 \%$ in surface cages and $95 \%$ in bottom cages.

\section{Diet effect on red abalone growth and survival}

Red abalone fed a $100 \% M$. pyrifera diet had a DISL of $88 \pm 10 \mu \mathrm{m} \cdot \mathrm{d}^{-1}$, a TG of $7.94 \pm 0.88 \mathrm{~mm}$, a mean MGR of $2.6 \pm 0.3 \mathrm{~mm} \cdot \mathrm{month}^{-1}$, and $99 \%$ survival. With the $100 \%$ E. arborea diet, DISL was $99 \pm 7 \mu \mathrm{m} \cdot \mathrm{d}^{-1}$, TG was $8.86 \pm 0.62 \mathrm{~mm}$, mean MGR was $3.0 \pm 0.2 \mathrm{~mm} \cdot \mathrm{month}^{-1}$, and survival was $98 \%$. Abalone fed a mixed diet of $50 \%$ M. pyrifera $150 \%$. porra had a DISL of $74 \pm 13 \mu \mathrm{m} \cdot \mathrm{d}^{-1}, \mathrm{TG}$ of $6.68 \pm 1.19 \mathrm{~mm}$, mean MGR of $2.2 \pm 0.4 \mathrm{~mm} \cdot \mathrm{month}^{-1}$, and $94 \%$ survival. Mean DISL and MGR rates for each cage are presented in Table 2 . We did not find statistically significant differences in the growth parameters among diet treatments (Fig. 4, Table 1; two-way ANOVA, $F_{2,4}=4.120, P=$ $0.107)$. We also carried out a power analysis to find the least significant sample size for the difference between the 3 diet treatments to be statistically significant. If our experimental design had had 2 or 3 replicates per treatment, the difference in growth between diets might have been statistically significant $(\alpha=0.05, \sigma=0.77, \delta=0.78): N=12$, power $=$ 0.74 ; and $N=18$, power $=0.94$, respectively. Mean growth across all treatments was $84 \pm 15 \mu \mathrm{m} \cdot \mathrm{d}^{-1}$ during the first sampling period (days $0-46$ ) and $90 \pm 9 \mu \mathrm{m} \cdot \mathrm{d}^{-1}$ during the second period (days 47-90) (Fig. 4).

\section{Temperature}

For the surface treatment, mean temperature was $13.69 \pm$ $0.92{ }^{\circ} \mathrm{C}(\max =18.62, \min =12.01)$ and for the bottom periodo (del día 47 al 90) (Fig. 3). La tasa de supervivencia promedio de los abulones fue de $99 \%$ en superficie y $95 \%$ en las jaulas de fondo.

\section{Efecto de la dieta en la supervivencia y el crecimiento del abulón rojo}

Los abulones alimentados con una dieta de 100\% M. pyrifera tuvieron un IDLC de $88 \pm 10 \mu \mathrm{m} \cdot \mathrm{d}^{-1}$, un CT de $7.94 \pm 0.88 \mathrm{~mm}$, una TCM promedio de $2.6 \pm 0.3 \mathrm{~mm} \cdot \mathrm{mes}^{-1}$ y una supervivencia del $99 \%$. Con la dieta de $100 \%$ E. arborea, se registró un IDLC de $99 \pm 7 \mu \mathrm{m} \cdot \mathrm{d}^{-1}$, un CT de $8.86 \pm 0.62 \mathrm{~mm}$, una TCM promedio de $3.0 \pm 0.2 \mathrm{~mm} \cdot \mathrm{mes}^{-1}$ y una supervivencia del 98\%. Los abulones alimentados con una dieta mixta de $50 \% P$. porra $/ 50 \% M$. pyrifera presentaron un IDLC de $74 \pm 13 \mu \mathrm{m} \cdot \mathrm{d}^{-1}$, un CT de $6.68 \pm$ $1.19 \mathrm{~mm}$, una TCM promedio de $2.2 \pm 0.4 \mathrm{~mm} \cdot \mathrm{mes}^{-1} \mathrm{y}$ una supervivencia del 94\%. Los promedios de IDLC y TCM por jaula se encuentran en la Tabla 2. Tampoco se encontraron diferencias estadísticamente significativas en los parámetros de crecimiento entre diferentes dietas (Fig. 4, Tabla 1, ANDEVA de 2 vías, $\left.F_{2,4}=4.120, P=0.107\right)$. De nuevo, llevamos a cabo un análisis de poder para hallar el menor tamaño de muestra que permitiría detectar diferencias estadísticas entre las 3 dietas. Si nuestro diseño experimental hubiese tenido 2 o 3 réplicas por tratamiento, la diferencia en crecimiento entre las dietas podría haber sido estadísticamente significativa ( $\alpha=0.05, \sigma=0.77, \delta=0.78)$ : $N=12$, poder $=0.74$; y $N=18$, poder $=0.94$, respectivamente. El crecimiento promedio fue de $84 \pm 15 \mu \mathrm{m} \cdot \mathrm{d}^{-1}$ durante el primer periodo de muestreo (del día 0 al 46) y de $90 \pm 9 \mu \mathrm{m} \cdot \mathrm{d}^{-1}$ durante el segundo periodo (del día 47 al 90) (Fig.4).

\section{Temperatura}

La temperatura promedio fue de $13.69 \pm 0.92{ }^{\circ} \mathrm{C}$ (máx $=$ $18.62, \min =12.01)$ en el tratamiento de superficie y de 14.01 $\pm 0.94{ }^{\circ} \mathrm{C}(\operatorname{máx}=17.85, \min =12.01)$ en el tratamiento de fondo (Fig. 5). No encontramos diferencias estadísticas significativas entre las temperaturas promedio diarias entre 
treatment, mean temperature was $14.01 \pm 0.94{ }^{\circ} \mathrm{C}(\max =$ 17.85, $\min =12.01$ ) (Fig. 5). Using daily means, there were no statistically significant differences in temperature between the surface and the bottom (ANOVA, $F_{1,270}=0.88, P=0.41$ ).

\section{Discussion}

To test the potential for red abalone, H. rufescens, mariculture at San Jeronimo Island, Baja California, Mexico, we explored the feasibility of growing red abalone in cages attached to a long-line system and assessed the effects of depth and diet on their growth. The present study describes the broad importance of developing experiments and analyses to provide alternative ways to enhance production and inform management of complex systems responding to complex climate change effects such as extreme marine heatwaves. Our results indicate that red abalone mariculture is feasible at San Jeronimo Island and that the use of varying culture depths and the use of diets with alternative algal species may be effective strategies to cope with the effects of marine heatwaves and storm surges. The power analyses showed that appropriate replication would have found slight differences between diets and depths and would have also allowed us to test for interactions between the variables. Therefore, given the lack of balanced treatment replication, due to logistic constraints, the results from this small-scale local experiment should be taken cautiously. el tratamiento de superficie y el de fondo (ANDEVA, $F_{1,270}=$ $0.88, P=0.41)$.

\section{Discusión}

Para investigar el potencial para la maricultura de abulón rojo, H. rufescens, en isla San Jerónimo, Baja California, México, exploramos la factibilidad de su cultivo en jaulas aseguradas a un sistema de línea madre e investigamos los efectos de la dieta y la profundidad en su crecimiento. El presente estudio describe la importancia de desarrollar experimentos y análisis con el fin de proponer maneras alternativas de optimizar la producción e informar el manejo de sistemas complejos ante múltiples efectos del cambio climático, tales como ondas de calor extremas. Nuestros resultados indican que la maricultura de abulón rojo es factible en isla San Jerónimo y que tanto el uso de diferentes profundidades de cultivo como el de dietas con especies alternativas de algas pueden ser estrategias efectivas para lidiar con los efectos de las ondas de calor y las tormentas. Los análisis de poder demostraron que una replicación adecuada podría haber encontrado diferencias sutiles entre dietas y profundidades y podría haber permitido, además, examinar la interacción entre estas variables. Por tanto, dada la falta de replicación balanceada de los tratamientos debido a limitaciones logísticas, los resultados de este experimento a pequeña escala deben interpretarse con precaución.

Table 2. Total mean growth in shell length of red abalone fed 3 different macroalgal diets in the surface and bottom treatments. DISL, daily increase in shell length; MGR, monthly growth rate.

Tabla 2. Crecimiento promedio total en longitud de concha del abulón rojo alimentado con 3 dietas de macroalgas diferentes en los tratamientos en superficie y en el fondo. DISL, incremento diario en longitud de la concha; MGR, tasa de crecimiento mensual.

\begin{tabular}{clcccc}
\hline Cage & \multicolumn{1}{c}{ Diet } & Depth & $\begin{array}{c}\text { DISL } \\
\left(\mu \mathrm{m} \cdot \mathrm{d}^{-1}\right)\end{array}$ & $\begin{array}{c}\text { MGR } \\
\left(\mathrm{mm} \cdot \mathrm{month}^{-1}\right)\end{array}$ & $\begin{array}{c}\text { Total growth } \\
(\mathrm{mm})\end{array}$ \\
\hline 1 & $100 \%$ Macrocystis pyrifera & Surface & 81 & 2.4 & 7.31 \\
2 & $100 \%$ Eisenia arborea & Surface & 103 & 3.1 & 9.30 \\
3 & $50 \%$ Macrocystis pyrifera, & Surface & 84 & 2.5 & 7.52 \\
& $50 \%$ Pelagophycus porra & & & & \\
4 & $100 \%$ Macrocystis pyrifera & Surface & 102 & 3.1 & 9.19 \\
5 & $100 \%$ Macrocystis pyrifera & Bottom & 81 & 2.4 & 7.31 \\
6 & $100 \%$ Eisenia arborea & Bottom & 94 & 2.8 & 8.43 \\
7 & $50 \%$ Macrocystis pyrifera, & Bottom & 65 & 1.9 & 5.84 \\
& $50 \%$ Pelagophycus porra & & & & \\
8 & $100 \%$ Macrocystis pyrifera & Bottom & 88 & 2.7 & 7.95 \\
& & Average & 87 & 2.6 & 7.90 \\
& & SD & 13 & 0.4 & 1.10 \\
& & Min & 65 & 1.9 & 5.80 \\
& & Max & 103 & 3.1 & 9.30 \\
\hline
\end{tabular}




\section{Depth effect on red abalone growth and survival}

We tested the effect of depth on the growth and survival of juvenile red abalone, as climatic changes such as sea surface warming and storms will likely affect growth and survivorship for future mariculture projects. There were no statistically significant differences in growth between depth treatments (Fig. 3, Table 1). However, as the power analysis showed, if we had had 3 or 4 replicates per treatment, we might have found statistically significant differences on growth with depth, with slightly higher growth in the surface cages, although probably not important from the aquaculture perspective, since cages would be lowered to the bottom only for short-term periods during storms. Temperature was similar between the surface water and at $5 \mathrm{~m}$ depth (Fig. 5), so the trend of slightly greater growth at the surface may have been related to other variables such as dissolved oxygen, $\mathrm{pH}$, light availability, currents, or turbidity (Morash and Alter 2016). We also noticed more sediment in the bottom cages, and this may have contributed to the trend of slightly lower growth rate and survival observed at this depth. However, we did not measure sedimentation rates, and their effects on abalone growth and survival in cage culture systems have not yet been studied to our knowledge. Our results indicate that during a severe storm in the area, it would be possible to lower the cages to protect the caging system from damage due to storm-generated waves and surge without negatively affecting abalone growth rates.

\section{Diet effect on red abalone growth and survival}

In our experiment, we found that red abalone tended to grow better with the $E$. arborea diet $\left(99 \mu \mathrm{m} \cdot \mathrm{d}^{-1}\right.$, $\left.3 \mathrm{~mm} \cdot \mathrm{month}^{-1}\right)$ than with the $M$. pyrifera diet $\left(88 \mu \mathrm{m} \cdot \mathrm{d}^{-1}\right.$, $2.6 \mathrm{~mm} \cdot \mathrm{month}^{-1}$ ) or the $50 \% \mathrm{P}$. porra $/ 50 \% \mathrm{M}$. pyrifera mixed diet $\left(75 \mu \mathrm{m} \cdot \mathrm{d}^{-1}, 2.2 \mathrm{~mm} \cdot \mathrm{month}^{-1}\right)$, although these differences were not significant (Fig. 4). However, as the power analysis showed, if we had been able to have 2 or 3 replicates per treatment, we would have found statistical differences on growth between diets, with slightly better growth with the E. arborea diet. Our results support the study by Zertuche-Gonzalez et al. (2014), who found similar growth rates in red abalone (similar size, $20 \mathrm{~mm}$ ) fed $M$. pyrifera $\left(2.5 \mathrm{~mm} \cdot \mathrm{month}^{-1}\right)$ and E. arborea $\left(2.2 \mathrm{~mm} \cdot \mathrm{month}^{-1}\right)$ in the same region. These slight differences in MGRs could be related to seed quality, temperature regime during culture, and the species-specific thermal preferences and optimum for growth. Díaz et al. (2000) found that juvenile red abalone $(46-59 \mathrm{~mm})$ acclimated to $17{ }^{\circ} \mathrm{C}$ preferred temperatures of $18.8^{\circ} \mathrm{C}$ and that optimum temperature for growth was $18.4{ }^{\circ} \mathrm{C}$. Steinarsson and Imsland (2003) examined the growth of abalone acclimated to $15^{\circ} \mathrm{C}$ when exposed to temperatures ranging from 11 to $22^{\circ} \mathrm{C}$ and reported optimal temperature to be $16.5^{\circ} \mathrm{C}$ for $21 \mathrm{~mm}$ red abalone and $17.2^{\circ} \mathrm{C}$ for 25-66 mm red abalone. Moreover, macroalgae morphotypes

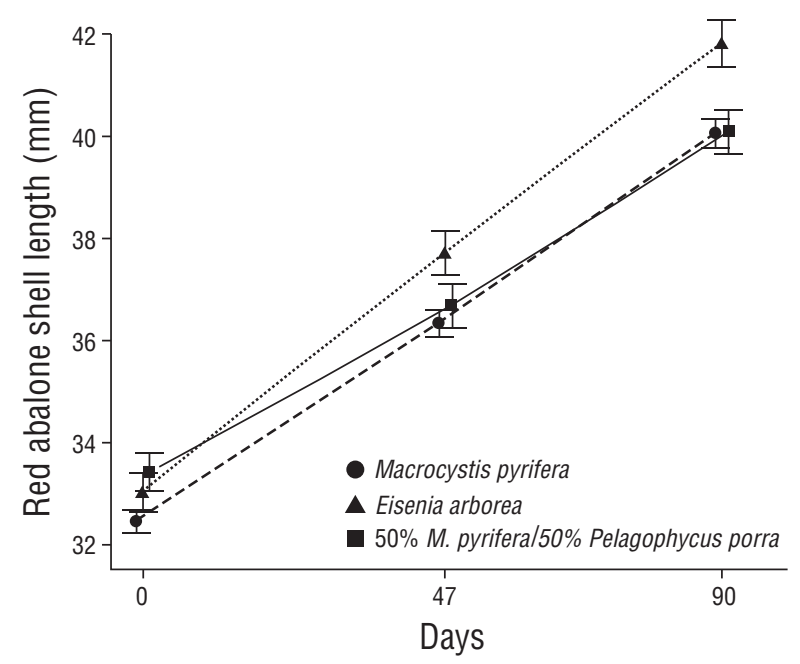

Figure 4. Red abalone, Haliotis rufescens, mean growth in shell length during $90 \mathrm{~d}$ of experiments fed with 3 different macroalgal diets (two-way analysis of variance, $F_{2,4}=4.120$, $P=0.107$ ): Macrocystis pyrifera diet, Eisenia arborea diet, 50\% M. pyrifera $/ 50 \%$ Pelagophycus porra mixed diet. Vertical bars denote standard error.

Figura 4. Crecimiento promedio en longitud de concha de abulón rojo, Haliotis rufescens, durante $90 \mathrm{~d}$ de experimento con 3 dietas de macroalgas diferentes (análisis de varianza de 2 vías, $F_{2,4}=$ 4.120, $P=0.107)$ : Macrocystis pyrifera, Eisenia arborea y dieta mixta $50 \%$ Pelagophycus porra $/ 50 \%$ M. pyrifera. Las barras verticales denotan el error estándar.

\section{Efecto de la profundidad en la supervivencia y el crecimiento del abulón rojo}

Examinamos el efecto de la profundidad en el crecimiento y la supervivencia de abulón rojo juvenil, dado que cambios en el clima, tales como el calentamiento de las aguas superficiales y tormentas más frecuentes e intensas, probablemente afecten el crecimiento y la supervivencia de organismos en proyectos futuros de maricultura. En este experimento no encontramos diferencias estadísticamente significativas en el crecimiento de los organismos entre profundidades (Fig. 3, Tabla 1). Sin embargo, y como demostró el análisis de poder, si hubiésemos tenido 3 o 4 réplicas por tratamiento, podríamos haber encontrado diferencias, con un crecimiento ligeramente mayor en las jaulas superficiales, aunque probablemente no suficientemente importante desde la perspectiva acuícola, ya que las jaulas sólo serían colocadas cerca del fondo por periodos cortos durante tormentas. La temperatura fue similar entre la superficie y el fondo a $5 \mathrm{~m}$ (Fig. 5), por lo que la tendencia a crecer ligeramente mejor en superficie debe estar relacionada con otras variables, tales como oxígeno disuelto, $\mathrm{pH}$, disponibilidad de luz, corrientes o turbidez (Morash y Alter 2016). También, notamos una mayor cantidad de sedimento en las jaulas profundas, lo cual puede haber contribuido a la tendencia de un crecimiento y 
and proximal composition can vary between sites in relation to oceanographic conditions such as currents, water motion, temperature, light, and nutrients (Roberson and Coyer 2004, Demes et al. 2009, Landa-Cansigno et al. 2017).

It is much easier and cheaper for fishers to obtain M. pyrifera than E. arborea. The latter does not create a floating canopy and requires scuba to harvest, whereas the canopy of $M$. pyrifera is easily harvested from the surface by hand from a boat. With future predictions of reduced wild populations of $M$. pyrifera in Baja California due to warmer temperatures or other environmental disturbances (BeasLuna et al. 2020), our results suggest, as previous studies, that $E$. arborea is a suitable alternative food source for red abalone aquaculture.

Growth rates in red abalone fed $M$. pyrifera have shown to range from $34 \mu \mathrm{m} \cdot \mathrm{d}^{-1}$ in organisms $8 \mathrm{~mm}$ shell length (Trevelyan et al. 1998) to $73 \mu \mathrm{m} \cdot \mathrm{d}^{-1}$ in organisms $20-60 \mathrm{~mm}$ shell length (Zertuche-González et al. 2014). The $88-\mu \mathrm{m} \cdot \mathrm{d}^{-1}$ growth rate observed in red abalone (32 $\mathrm{mm}$ shell length) fed with the $M$. pyrifera diet in this study is promising. Our findings suggest that San Jeronimo Island has high potential for red abalone mariculture based on an $M$. pyrifera diet alone, since currently it is the most abundant macroalga in the region. We examined alternatives to $M$. pyrifera algal diets that could be used for abalone in Baja California during extreme temperature events (Ladah y Zertuche 1999, Cavanaugh et al. 2019). Some authors recommend using red algae to boost red abalone growth; for example, Evans and Langdon (2000) obtained $124 \mu \mathrm{m} \cdot \mathrm{d}^{-1}$ in juvenile $H$. rufescens fed dulse Palmaria mollis. In contrast, Leighton (1966) demonstrated that $H$. rufescens had a distinct preference for brown algae, particularly $M$. pyrifera and E. menziesii. Other studies have used mixtures of brown algae; for example, una tasa de supervivencia ligeramente menor asociadas con la profundidad. Sin embargo, no medimos la tasa de sedimentación, y su posible efecto en el crecimiento y la supervivencia de abulones en jaulas no ha sido estudiado hasta la fecha, a nuestro conocimiento. Nuestros resultados indicaron que, durante tormentas severas en el área, sería posible descender las jaulas para proteger el sistema de daños debidos a las olas, sin afectar la tasa de crecimiento de los abulones.

\section{Efecto de la dieta en la supervivencia y el crecimiento del abulón rojo}

En nuestro experimento, hallamos que el abulón rojo tiene una tendencia a crecer mejor alimentándose de $E$. arborea $\left(99 \mu \mathrm{m} \cdot \mathrm{d}^{-1}, 3 \mathrm{~mm} \cdot \mathrm{mes}^{-1}\right)$ que de $M$. pyrifera $\left(88 \mu \mathrm{m} \cdot \mathrm{d}^{-1}\right.$, $2.6 \mathrm{~mm} \cdot \mathrm{mes}^{-1}$ ) o de $P$. porra mezclada con $M$. pyrifera (75 $\mu \mathrm{m} \cdot \mathrm{d}^{-1}, 2.2 \mathrm{~mm} \cdot \mathrm{mes}^{-1}$ ), aunque estas diferencias no fueron significativas (Fig. 4). Sin embargo, como demostró el análisis de poder, de haber tenido 2 o 3 réplicas por tratamiento, podríamos haber hallado diferencias en el crecimiento de los organismos según su dieta, con un crecimiento ligeramente mejor alimentándose de E. arborea. Nuestros resultados respaldan el estudio de Zertuche-Gonzalez et al. (2014), quienes hallaron tasas de crecimiento similares en abulones rojos (con tamaños similares de $20 \mathrm{~mm}$ ) alimentados de $M$. pyrifera $\left(2.5 \mathrm{~mm} \cdot \mathrm{mes}^{-1}\right)$ y $E$. arborea $\left(2.2 \mathrm{~mm} \cdot \mathrm{mes}^{-1}\right)$ en la misma región. Las ligeras diferencias en TCM pueden estar relacionadas a la calidad de la semilla, la temperatura durante el cultivo, y las preferencias termales específicas de crecimiento óptimo de la especie. Díaz et al. (2000) hallaron que los abulones rojos aclimatizados a $17^{\circ} \mathrm{C}$ tienen una temperatura preferencial de $18.8^{\circ} \mathrm{C}$ y una temperatura óptima de crecimiento de $18.4^{\circ} \mathrm{C}$. Por otro lado,

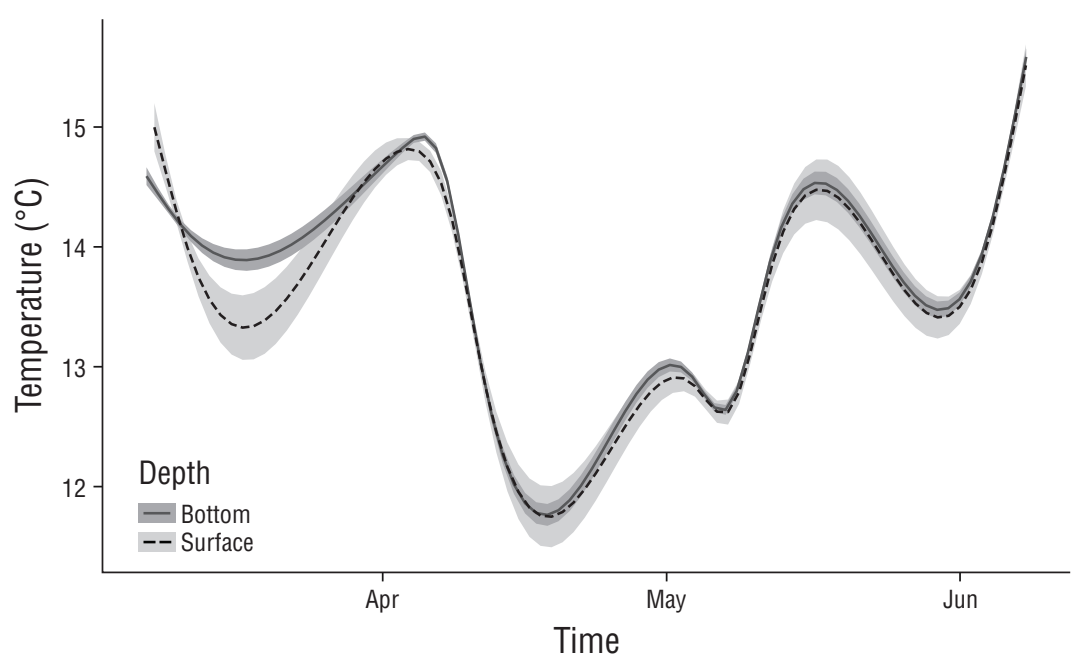

Figure 5. Recorded temperature $\left({ }^{\circ} \mathrm{C}\right)$ in the depth treatments during the experimental period. Grey shades indicate 0.95 confidence intervals. Figura 5. Temperatura registrada $\left({ }^{\circ} \mathrm{C}\right)$ en los tratamientos de profundidad durante el experimento. La sombras grises denotan el intervalo de confianza de 0.95 . 
Searcy-Bernal and Salas-Garza (1990) obtained a growth rate of $74 \mu \mathrm{m} \cdot \mathrm{d}^{-1}$ in H. rufescens fed both M. pyrifera and E. menziesii. Abalone growth rates on natural diets are reported to range from $0.8 \mu \mathrm{m} \cdot \mathrm{d}^{-1}$ for Haliotis iris (20 mm shell length) fed Ulva lactuca (Stuart and Brown 1994) to $139 \mu \mathrm{m} \cdot \mathrm{d}^{-1}$ for Haliotis discuss hannai (24-34 mm shell length) fed Eisenia bicyclis (Uki et al. 1986). More studies are needed to understand the effect of mixed algae diets and their economic viability in mariculture systems. Also, in order to develop an optimum mariculture system in a particular region, future research should consider that feeding rates of abalone depend on body size, type of food, density, and temperature (Capinpin et al. 1999, Nelson et al. 2002).

\section{Temperature}

Temperature is the main variable that controls the rates of most metabolic processes in abalone (Rogers-Bennett et al. 2010, Morash and Alter 2016). Outside their optimum range, individuals adjust basic physiological functions to maintain basal metabolic demands (Medina-Romo et al. 2010). In red abalone, preferential temperature increases during development until $30 \mathrm{~mm}$ shell length and then declines as the individual grows (Steinarsson and Imsland 2003). The preferential temperature of $H$. rufescens is $18{ }^{\circ} \mathrm{C}$ (Leighton 1974, Díaz et al. 2000). At $25^{\circ} \mathrm{C}$ H. rufescens begins to present detachment symptoms, and the maximum critical temperature is $27.5^{\circ} \mathrm{C}$ (Díaz et al. 2006). In this study, the mean temperature of the surface treatment was $13.69 \pm 0.92{ }^{\circ} \mathrm{C}(\max =18.62, \min =12.01)$ and for the bottom treatment it was $14.01 \pm 0.94{ }^{\circ} \mathrm{C}(\max =17.85$, min $=12.01$ ). Temperature and $H$. rufescens growth and survival data indicate that San Jeronimo Island has high potential for red abalone mariculture, at least during the months over which this experiment was conducted. It is critical to have a robust understanding of the effect of temperature on abalone metabolism and growth (Morash and Alter 2016) to predict the future effects of rising ocean temperature on abalone farming.

\section{Future directions}

It is of utmost importance to develop sustainable mariculture in Mexico, as it will diversify the activities of fishing communities, produce valuable products, and help restore and conserve marine species. For example, abalone aquaculture and mariculture projects have been very successful in several Asian countries. Currently, 155,939 mt of abalone are produced in China, South Korea, and Japan, equivalent to $95 \%$ of the world abalone production (Cook 2019). Valuable insights from these cases can be applied to future efforts within Mexico. For example, in these countries, aquaculture is co-managed between academia, private initiatives, local communities, and government agencies (Lee 2019). Conservation aquaculture projects in Baja California have the
Steinarsson e Imsland (2003) examinaron el crecimiento de abulones aclimatizados a $15^{\circ} \mathrm{C}$, y expuestos a temperaturas con un intervalo de 11 a $22^{\circ} \mathrm{C}$, y reportaron una temperatura óptima de $16.5^{\circ} \mathrm{C}$ para abulones rojos de $21 \mathrm{~mm}$ y de $17.2^{\circ} \mathrm{C}$ para abulones rojos de 25-66 mm. Además, también hay que considerar que los morfotipos y la composición proximal de las macroalgas pueden variar entre sitios, relacionándose con condiciones oceanográficas como corrientes, movimiento del agua, temperatura, luz y nutrientes (Roberson y Coyer 2004, Demes et al. 2009, Landa-Cansigno et al. 2017).

Es mucho más sencillo y barato obtener $M$. pyrifera que E. arborea, ya que esta última no crea un dosel flotante. Se requiere de buceo autónomo para recolectar E. arborea, mientras que $M$. pyrifera puede ser recolectada fácilmente a mano desde un bote. Simultáneamente, y tomando en consideración las predicciones futuras de una reducción de las poblaciones silvestres de M. pyrifera en Baja California debido a temperaturas más altas y otros estresores (BeasLuna et al. 2020), nuestros resultados sugieren que el uso de E. arborea es una alternativa adecuada como fuente de alimento en el cultivo de abulón rojo.

Las tasas de crecimiento del abulón rojo alimentado con M. pyrifera varían de $34 \mu \mathrm{m} \cdot \mathrm{d}^{-1}$ en organismos de longitud de concha de $8 \mathrm{~mm}$ (Trevelyan et al. 1998) a $73 \mu \mathrm{m} \cdot \mathrm{d}^{-1}$ en organismos de longitud de concha de 20-60 mm (ZertucheGonzález et al. 2014). La tasa de crecimiento de $88 \mu \mathrm{m} \cdot \mathrm{d}^{-1}$ observada en abulón rojo (32 $\mathrm{mm}$ de longitud de concha) alimentado con M. pyrifera en este estudio es prometedor. Nuestros resultados sugieren que isla San Jerónimo tiene un potencial alto para la maricultura del abulón rojo basado simplemente en una dieta de M. pyrifera, ya que actualmente es la macroalga más abundante en la región. Examinamos dietas alternativas a $M$. pyrifera para abulón que pudiesen ser utilizadas en Baja California durante eventos de temperaturas extremas (Ladah y Zertuche 1999, Cavanaugh et al. 2019). Algunos autores recomiendan el uso de algas rojas para aumentar el crecimiento de abulón rojo; por ejemplo, Evan y Langdon (2000) obtuvieron un crecimiento de $124 \mu \mathrm{m} \cdot \mathrm{d}^{-1}$ en juveniles de $H$. rufescens alimentados con Palmaria mollis. En contraste, Leighton (1966) demostró que $H$. rufescens tiene una clara preferencia por las algas pardas, M. pyrifera y E. menziesii en particular. Otros estudios han utilizado mezclas de algas pardas; por ejemplo, Searcy-Bernal y Salas-Garza (1990) obtuvieron una tasa de crecimiento de $74 \mu \mathrm{m} \cdot \mathrm{d}^{-1}$ en $H$. rufescens alimentados con $M$. pyrifera y E. menziesii. Las tasas de crecimiento de abulón con una dieta natural se reportan con un intervalo de $0.8 \mu \mathrm{m} \cdot \mathrm{d}^{-1}$ para Haliotis iris (20 $\mathrm{mm}$ de longitud de concha) alimentado con Ulva lactuca (Stuart y Brown 1994) hasta $139 \mu \mathrm{m} \cdot \mathrm{d}^{-1}$ para Haliotis discus hannai (24-34 mm de longitud de concha) alimentado con Eisenia bicyclis (Uki et al. 1986). Más estudios son necesarios para entender el efecto de una dieta de algas mixta y su viabilidad económica en sistemas de maricultura. Es vital que investigaciones futuras consideren que las tasas de alimentación dependen del tamaño del abulón, 
potential to enhance the resiliency of coastal communities to the impacts of climate change. Although the small-scale mariculture system used in this experiment was successful at San Jeronimo Island, more research should be conducted to determine its upscaling potential and feasibility at other locations.

\section{ACKNOWLEDGMENTS}

This paper was prepared with funds provided by the Mexican Secretary of Public Education (UABC-PTC-669), Universidad Autónoma de Baja California (240-21a. CONV INT), and the National Council for Science and Technology (Mexico, \#CVU: 860611). We thank the fishing cooperative "Ensenada," the NGO "Comunidad y Biodiversidad", and the group "Monitoreo y Conservación de Especies" for their hard work in developing the mariculture system and feeding the abalone, and Mex-Cal (www.mex-cal.org) for their time and effort providing feedback and assistance in the field. We thank COBI-UC Davis Phase II-01112018-28022020. We also thank Sarah J Teck and 2 anonymous reviewers for their helpful suggestions to improve the manuscript and Lucia M Rodriguez for her help with the translation.

\section{REFERENCES}

Aitkin M. 1978. The analysis of unbalanced cross-classifications. Royal Statistical Society. 141(2):195-223. https://doi.org/10.2307/2344453

Arafeh-Dalmau N, Montaño-Moctezuma G, Martínez JA, BeasLuna R, Schoeman DS, Torres-Moye G. 2019. Extreme Marine Heatwaves alter kelp forest community near its equatorward distribution limit. Frontiers in Marine Science. 6:499. https://doi.org/10.3389/fmars.2019.00499

Arafeh-Dalmau N, Schoeman DS, Montaño-Moctezuma G, Micheli F, Rogers-Bennett L, Olguin-Jacobson C, Possingham HP. 2020. Marine heatwaves threaten kelp forests. Science Letters. 367(6478):635. https://doi.org/10.1126/science.aba5244

Barange M, Perry RI. 2009. Physical and ecological impacts of climate change relevant to marine and inland capture fisheries and aquaculture. In: Cochrane K, De Young C, Soto D, Bahri T. (eds.), Climate Change Implications for Fisheries and Aquaculture. Overview of Current Scientific Knowledge. Rome (Italy): FAO. p. 7-106.

Beas-Luna R, Micheli F, Woodson CB, Carr M, Malone D, Torre J, Boch CA, Caselle JE, Edwards M, Freiwald J, et al. 2020. Geographic variation in responses of kelp forest communities of the California Current to recent climatic changes. Glob Change Biol. 26(11):6457-6473.

https://doi.org/10.1111/gcb.15273

Ben-Horin T, Lafferty KD, Bidegain G, Lenihan HS. 2016. Fishing diseased abalone to promote yield and conservation. Phil Trans Soc B. 371:(1689):20150211. https://doi.org/10.1098/rstb.2015.0211

Boch CA, Micheli F, AlNajjar M, Monismith SG, Beers JM, Bonilla JC, Espinoza AM, Vazquez-Vera L, Woodson CB. 2018. Local oceanographic variability influences the performance of juvenile abalone under climate change. Sci Rep. 8(1):5501. https://doi.org/10.1038/s41598-018-23746-Z tipo de alimento, densidad de organismos y temperatura del medio (Capinpin et al. 1999, Nelson et al. 2002) si se busca desarrollar un sistema de maricultura optimizado para cada región.

\section{Temperatura}

La temperatura es la variable principal que controla la tasa de la mayoría de los procesos metabólicos en el abulón (Rogers-Bennett et al. 2010, Morash y Alter 2016). Fuera de su intervalo óptimo, los individuos ajustan funciones fisiológicas para mantener sus demandas metabólicas basales (Medina-Romo et al. 2010). En el abulón rojo, la temperatura preferencial aumenta durante el desarrollo hasta la talla de $30 \mathrm{~mm}$ de longitud de concha, y empieza a decrecer conforme crece el organismo (Steinarsson e Imsland 2003). La temperatura preferencial para $H$. rufescens es de $18{ }^{\circ} \mathrm{C}$ (Leighton 1974, Díaz et al. 2000). A los $25^{\circ} \mathrm{C}, H$. rufescens empieza a presentar síntomas de desprendimiento, y la temperatura máxima crítica es $27.5^{\circ} \mathrm{C}$ (Díaz et al. 2006). En este estudio, la temperatura promedio del tratamiento de superficie fue de $13.69 \pm 0.92{ }^{\circ} \mathrm{C}(\max =18.62, \min =12.01)$ y la del tratamiento de fondo fue de $14.01 \pm 0.94^{\circ} \mathrm{C}(\max =17.85, \min =$ 12.01). La temperatura y los datos de crecimiento y supervivencia indican que isla San Jerónimo tiene un alto potencial para la maricultura de abulón rojo, al menos durante los meses en los cuales se condujo este experimento. Es crítico obtener una comprensión robusta del efecto de la temperatura en el metabolismo y el crecimiento del abulón (Morash y Alter 2016) para predecir los efectos futuros de las temperaturas oceánicas en el cultivo del mismo.

\section{Direcciones futuras}

El desarrollo de la maricultura sostenible en México es de extrema importancia, ya que diversificará las actividades de las comunidades pesqueras, proporcionará productos valiosos y ayudará a la restauración y conservación de especies marinas. Por ejemplo, la acuicultura de abulón y los proyectos de maricultura han sido muy exitosos en varios países asiáticos. Actualmente, 155,939 tm de abulón son producidas en China, Corea del Sur y Japón, equivalente al 95\% de la producción mundial de abulón (Cook 2019). De estos casos, se pueden aplicar lecciones valiosas para futuros esfuerzos en México. Por ejemplo, en estos países, la acuicultura es co-manejada entre la academia, las iniciativas privadas, las comunidades locales y las agencias gubernamentales (Lee 2019). Los proyectos de acuicultura de la conservación en Baja California tienen el potencial de fortalecer la resiliencia de comunidades costeras a los impactos del cambio climático. Aunque el sistema de maricultura de pequeña escala utilizado en este experimento fue exitoso en isla San Jerónimo, se necesita investigación adicional para determinar su potencial de expansión y factibilidad en otras localidades. 
Brown MB, Forsythe AB. 1974. Robust tests for the equality of variances. J American Statistical Association. 69(346):364367.

Capinpin EC, Toledo JD, Encena VC, Doi M. 1999. Density dependent growth of the tropical abalone Haliotis asinina in cage culture. Aquaculture. 171(3-4):227-235.

https://doi.org/10.1016/s0044-8486(98)00490-6

Cavanaugh KC, Reed DC, Bell TW, Castorani MCN, Beas-Luna R. 2019. Spatial variability in the resistance and resilience of giant kelp in southern and Baja California to a multiyear heatwave. Frontiers in Marine Science. 6:1-14. https://doi.org/10.3389/fmars.2019.00413

Cook PA. 2016. Recent trends in worldwide abalone production. J Shellfish Res. 35(3):581-583. https://doi.org/10.2983/035.035.0302

Cook PA. 2019. Worldwide abalone production statistics. J Shellfish Res. 38(2):401-404. https://doi.org/10.2983/035.038.0222

Demes KW, Graham MH, Suskiewicz TS. 2009. Phenotypic plasticity reconciles incongruous molecular and morphological taxonomies: the giant kelp, Macrocystis (Laminariales, Phaeophyceae), is a monospecific genus. Phycological Society of America. 45:1266-1269. https://doi.org/10.1111/j.1529-8817.2009.00752.x

Díaz F, del Río-Portílla MA, Sierra E, Aguilar M, Re-Araujo AD. 2000. Preferred temperature and critical thermal maxima of red abalone Haliotis rufescens. J Therm Biol. 25(3):257-261. https://doi.org/10.1016/s0306-4565(99)00032-7

Díaz F, Re AD, Medina Z, Re G, Valdez G, Valenzuela F. 2006. Thermal preference and tolerance of green abalone Haliotis fulgens (Philippi, 1845) and pink abalone Haliotis corrugata (Gray, 1828). Aquaculture Research. 37(9):877-884. https://doi.org/10.1111/j.1365-2109.2006.01506.x

[DOF] Diario Oficial de la Federación. 2018. Carta Nacional Pesquera. Mexico: Secretaría de Agricultura, Ganadería, Desarrollo Rural, Pesca y Alimentación.

Evans F, Langdon CJ. 2000. Co-culture of dulse Palmaria mollis and red abalone Haliotis rufescens under limited flow conditions. Aquaculture. 185(1-2):137-158. https://doi.org/10.1016/s0044-8486(99)00342-7

Froehlich HE, Gentry RR, Halpern BS. 2017. Conservation aquaculture: shifting the narrative and paradigm of aquaculture's role in resource management. Biol Conserv. 215:162-168. https://doi.org/10.1016/j.biocon.2017.09.012

Froehlich HE, Gentry RR, Halpern BS. 2018. Global change in marine aquaculture production potential under climate change. Nat Ecol Evol. 2(11):1745-1750.

https://doi.org/10.1038/s41559-018-0669-1

Garcia-Esquivel Z, Felbeck H. 2009. Comparative performance of juvenile red abalone, Haliotis rufescens, reared in laboratory with fresh kelp and balanced diets. Aquaculture Nutrition. 15(2):209-217. https://doi.org/10.1111/j.1365-2095.2008.00585.x

Guzmán-del Próo SA, Carrillo-Laguna J, Belmar-Pérez J, MuciñoDíaz M, Sierra-Rodríguez P. 2013. Time series of juvenile and adult green abalone (Haliotis fulgens) in Bahía Tortugas, Mexico: its potential application as a forecast of future stock abundance. J Shellfish Res. 32(1):217-221. https://doi.org/10.2983/035.032.0128

Guzmán-del Próo SA, del Monte-Luna P. 2017. Abalone reef productivity and the problem of scale in the management of the mexican abalone fishery. Ocean and Coastal Management. 144:1-6.

https://doi.org/10.1016/j.ocecoaman.2017.04.005

\section{Agradecimientos}

Este artículo fue preparado con fondos provistos por la Secretaría de Educación Pública (México, UABC-PTC-669), la Universidad Autónoma de Baja California (240-21a. CONV INT) y el Consejo Nacional de Ciencia y Tecnología (México, \#CVU: 860611). Agradecemos a la cooperativa pesquera "Ensenada", a la asociación civil "Comunidad y Biodiversidad" (COBI) y al grupo "Monitoreo y Conservación de Especies" su arduo trabajo para desarrollar el sistema de maricultura y alimentar a los abulones, y a Mex-Cal (www.mex-cal.org) su tiempo y esfuerzo con la retroalimentación y en el campo. Agradecemos a COBI-UC Davis Fase II-01112018-28022020. También agradecemos a Sarah J Teck y a 2 revisores anónimos por sus útiles sugerencias para mejorar el presente manuscrito y a Lucía M Rodriguez por su ayuda con la traduccion del mismo.

Hernández-Carmona G, García O, Robledo D, Foster M. 2000. Restoration techniques for Macrocystis pyrifera (Phaeophyceae) populations at the southern limit of their distribution in México. Botanica Marina. 43(3):274-284. https://doi.org/10.1515/bot.2000.029

Hernández-Carmona G, Robledo D, Serviere-Zaragoza E. 2001. Effect of nutrient availability on Macrocystis pyrifera recruitment and survival near its southern limit off Baja California. Botanica Marina. 44(3):221-229. https://doi.org/10.1515/BOT.2001.029

Hopkins KD. 1992. Reporting fish growth: a review of the basics. J World Aquacult Soc. 23(3):173-179.

Karpov KA, Haaker PL, Taniguchi IK, Rogers-Bennett L. 2000. Serial depletion and the collapse of the California abalone (Haliotis spp.) fishery. Can Spec Publ Fishe Aquat Sci. 11-24.

Ladah LB, Zertuche-González JA, Hernández-Carmona G. 1999. Giant kelp (Macrocystis pyrifera, Phaeophyceae) recruitment near its southern limit in Baja California after mass disappearance during ENSO 1997-1998. J Phycol. 35(6):11061112 . https://doi.org/10.1046/j.1529-8817.1999.3561106.x

Lafferty KD, Kuris AM. 1993. Mass mortality of abalone Haliotis cracherodii on the California Channel Islands: tests of epidemiological hypotheses. Mar Ecol Prog Ser. 96(3):239-248. https://doi.org/10.3354/meps096239

Landa-Cansigno C, Hernández-Carmona G, Arvizu-Higuera DL, Muñoz-Ochoa M, Hernández-Guerrero CJ. 2017. Bimonthly variation in the chemical composition and biological activity of the brown seaweed Eisenia arborea (Laminariales: Ochrophyta) from Bahía Magdalena, Baja California Sur, Mexico. J App Phycol. 29(5):2605-2615.

https://doi.org/10.1007/s10811-017-1195-2

Langsrud Ø. 2003. ANOVA for unbalanced data: use Type II instead of Type III sums of squares. Statistics and Computing. 13:163-167.

Lee SG. 2019. Marine stock enhancement, restocking, and sea ranching in Korea. In: Kideghesho J, Rija A (eds.), Wildlife Management - Failures, Successes and Prospects: IntechOpen. p. 125-139. https://doi.org/10.5772/intechopen.78373 
Leighton DL. 1966. Studies of food preferences in algivorous invertebrates of southern California kelp beds. Pac Sci. 20(1):104-113.

Leighton DL. 1974. The influence of temperature on larval and juvenile growth in three species of southern California abalones. Fish Bull. 72(4):1137-1145.

Lonhart SI, Jeppesen R, Beas-Luna R, Crooks JA, Lorda J. 2019. Shifts in the distribution and abundance of coastal marine species along the eastern Pacific Ocean during marine heatwaves from 2013 to 2018. Mar Biodivers Rec. 12(1):13. https://doi.org/10.1186/s41200-019-0171-8

Medina-Romo Z, Re AD, Díaz F, Mena A. 2010. Physiological responses of pink abalone Haliotis corrugata (Gray, 1828) exposed to different combinations of temperature and salinity. Aquac Res. 41(7):953-960. https://doi.org/10.1111/j.1365-2109.2009.02377.x

Micheli F, Saenz-Arroyo A, Greenley A, Vazquez L, EspinozaMontes JA, Rossetto M, de Leo GA. 2012. Evidence that marine reserves enhance resilience to climatic impacts. PLoS ONE. 7(7): e40832. https://doi.org/10.1371/journal.pone.0040832

Morales-Bojórquez E, Muciño-Díaz MO, Vélez-Barajas JA. 2008. Analysis of the decline of the abalone fishery (Haliotis fulgens and $H$. corrugata) along the Westcentral coast of the Baja California Peninsula, Mexico. J Shellfish Res. 27(4);865-870. https://doi.org/10.2983/0730-8000(2008)27[865:aotdot]2.0.co;2

Morash AJ, Alter K. 2016. Effects of environmental and farm stress on abalone physiology: perspectives for abalone aquaculture in the face of global climate change. Reviews in Aquaculture. 8(4):342-368. https://doi.org/10.1111/raq.12097

Nelson MM, Leighton DL, Phleger CF, Nichols PD. 2002. Comparison of growth and lipid composition in the green abalone, Haliotis fulgens, provided specific macroalgal diets. Comparative Biochemistry and Physiology Part B: Biochemistry and Molecular Biology. 131(4):695-712. https://doi.org/10.1016/s1096-4959(02)00042-8

Oliver ECJ, Donat MG, Burrows MT, Moore PJ, Smale DA, Alexander LV, Benthuysen JA, Feng M, Gupta AS, Hobday AJ, et al. 2018. Longer and more frequent marine heatwaves over the past century. Nat Commun. 9:1324. https://doi.org/10.1038/s41467-018-03732-9

Roberson LM, Coyer JA. 2004. Variation in blade morphology of the kelp Eisenia arborea: incipient speciation due to local water motion? Mar Ecol Prog Ser. 282:115-128. https://doi.org/10.3354/meps282115
Rogers-Bennett L, Catton CA. 2019. Marine heat wave and multiple stressors tip bull kelp forest to sea urchin barrens. Sci Rep. 9(1):15050. https://doi.org/10.1038/s41598-019-51114-y

Rogers-Bennett L, Dondanville RF, Moore JD, Vilchis LI. 2010. Response of red abalone reproduction to warm water, starvation, and disease stressors: implications of ocean warming. J Shellfish Res. 29(3):599-611. https://doi.org/10.2983/035.029.0308

Searcy-Bernal R, Ramade-Villanueva MR, Altamira B. 2010. Current status of abalone fisheries and culture in Mexico. J Shellfish Res. 29(3):573-576. https://doi.org/10.2983/035.029.0304

Searcy-Bernal R, Salas-Garza AE. 1990. Investigaciones sobre el cultivo de Abulón en la Universidad Autónoma de Baja California, México. Serie Científica. UABC. 44-50.

Steinarsson A, Imsland AK. 2003. Size dependent variation in optimum growth temperature of red abalone (Haliotis rufescens). Aquaculture. 224(1-4):353-362. https://doi.org/10.1016/s0044-8486(03)00241-2

Stuart MD, Brown MT. 1994. Growth and diet of cultivated blackfooted abalone, Haliotis iris (Martyn). Aquaculture. 127(4):329-337. https://doi.org/10.1016/0044-8486(94)90235-6

Trevelyan GA, Mendoza JL, Buckley B. 1998. Increasing the yield of red abalone with the alga, Microcladia coulteri. J Shellfish Res. 17:631-633.

[UN-DESA] United Nations-Department of Economics and Social Affairs. 2018. The Sustainable Development Goals Report 2018. New York: UN.

Uki N, Sugiura M, Watanabe T. 1986. Dietary value of seaweeds occurring on the Pacific coast of Tohoku for growth of the abalone Haliotis discus hannai. Bull Jpn Soc Sci Fish. 52:257-266. https://doi.org/10.2331/suisan.52.257

Viera MP, Viçose GC, Fernández-Palacios H, Izquierdo M. 2014. Grow-out culture of abalone Haliotis tuberculata coccinea Reeve, fed land-based IMTA produced macroalgae, in a combined fish/abalone offshore mariculture system: effect of stocking density. Aquacult Res. 47(1):71-81. https://doi.org/10.1111/are.12467

Zertuche-González JA, Sánchez-Barredo M, Guzmán-Calderón JM, Altamirano-Gómez Z. 2014. Eisenia arborea J.E. Areschoug as abalone diet on an IMTA farm in Baja California, México. J Appl Phycol. 26(2):957-960. https://doi.org/10.1007/s10811-013-0138-9

Received February 2020, accepted November 2020. 